\title{
Malformações congênitas e uso de agrotóxicos no município de Giruá, RS
}

\author{
Congenital malformations and use of pesticides in the city of Giruá, $R S$
}

Luis Fernando Ferreira', Angélica Reolon da Costa', Silvana Ceolin $\mathbf{1}$

DOI: $10.1590 / 0103-1104202012615$

RESUMO O objetivo deste estudo foi relacionar o uso de agrotóxicos com a ocorrência de malformações congênitas no município de Giruá (RS), bem como determinar os fatores de risco associados. A pesquisa é de abordagem quantitativa, do tipo descritivo-exploratória, realizada durante o primeiro semestre de 2019. O estudo foi conduzido em duas etapas: (a) associação da ocorrência de malformações com o uso de agrotóxicos, que foi calculada por meio dos Odds Ratios, sendo o Intervalo de Confiança (IC) adotado para as amostras de 95\%; e (b) determinação dos fatores de risco associados, em que foi realizada uma entrevista com agricultores residentes no município. Percebe-se que as taxas de malformações congênitas do município de Giruá são superiores à média do estado para o mesmo período. Há probabilidade de que a ocorrência de malformações no município esteja associada ao uso de agrotóxicos, pois todos os valores dos Odds Ratios foram maiores que um. Os fatores de risco identificados foram baixa escolaridade, classe toxicológica e princípios ativos, uso inadequado de Equipamentos de Proteção Individual (EPIs), número de pessoas com diagnóstico de intoxicação ou que relataram algum sinal ou sintoma associado e armazenamento dos produtos.

PALAVRAS-CHAVE Agroquímicos. Anormalidades Congênitas. Saúde pública.

\begin{abstract}
The objective of this study was to relate the use of pesticides to the occurrence of congenital malformations in the city of Giruá (RS), as well as to determine the associated risk factors. The research has a quantitative, descriptive-exploratory approach, and was carried out during the first semester of 2019. The study was conducted in two stages: (a) association of the occurrence of malformations with the use of pesticides which was calculated using the Odds Ratios, with the Confidence Interval (CI) being adopted for the $95 \%$ samples; and (b) determination of the associated risk factors, for which an interview was conducted with farmers living in the municipality. It is noticed that the rates of congenital malformations in the city of Giruá, are higher than the state average for the same period. There is a probability that the occurrence of malformations in the municipality is associated with the use of pesticides, because all Odds Ratios were greater than one. The risk factors identified were low educational level, toxicological class and active principles, inadequate use of Personal Protective Equipment (PPE), number of people diagnosed with intoxication or who reported some associated signal or symptom and storage of the products.
\end{abstract}

KEYWORDS Agrochemicals. Congenital abnormalities. Public health.

1 Sociedade Educacional Três de Maio (Setrem) Três de Maio (RS), Brasil. angelicacosta@setrem. com.br 


\section{Introdução}

O modelo de produção capitalista e a exploração do meio ambiente foram um marco histórico na estrutura fundiária do Brasil, em prejuízo da agricultura sustável, agroecológica e orgânica'. Esse modelo impulsionou o uso e o registro de novos agrotóxicos no Brasil, colocando o País no ranking dos que mais utilizam esses produtos no mundo ${ }^{2}$. Esse cenário de utilização de múltiplas classes de agrotóxicos nos sistemas produtivos no Brasil tem trazido graves problemas para a saúde humana e ambiental ${ }^{3}$.

Na saúde, o uso de agrotóxicos está associado ao câncer, a alergias, distúrbios gastrointestinais, respiratórios, endócrinos, reprodutivos, neurológicos e risco de suicídio 4 . O uso de agrotóxicos está associado, ainda, à ocorrência de malformações fetais, como: testículo não descido, malformações do aparelho circulatório, sistema nervoso, aparelho digestivo, aparelho geniturinário, aparelho osteomuscular, anomalias cromossômicas, espinha bífida, malformações de fenda labial e palatina e outras malformações congênitas ${ }^{5,6}$.

Conforme dados epidemiológicos do Sistema de Informações sobre Nascidos Vivos (Sinasc), para a Unidade da Federação e para o estado do Rio Grande do Sul, as taxas de malformações congênitas, no período de 1999 a 2016, tiveram uma média de 6,45 e 8,21, respectivamente ${ }^{7}$. Na literatura nacional, existem poucas pesquisas associando o uso de agrotóxicos com a ocorrência de malformações fetais. A maioria é restrita aos estados do Mato Grosso e do Paraná.

A associação entre a ocupação paterna na agricultura e óbitos fetais, em que as famílias em áreas de maior utilização de agrotóxicos tiveram maior risco de viver gestações com morte fetal, incluindo as causadas por anomalias congênitas, foi evidenciada por Rigotto et al.8. Já as chances de $100 \%$ de maior ocorrência de malformação congênita entre as crianças cujas mães foram expostas durante o período periconcepcional a agrotóxicos, em relação às mães que não foram expostas, foram descritas por Oliveira et al. ${ }^{9}$, corroborando Dutra; Ferreira ${ }^{5,6}$.

Nesse contexto, ressalta-se a importância da atuação dos profissionais da saúde no desenvolvimento de práticas de cuidado e gestão aos grupos populacionais com risco de exposição aos agrotóxicos ${ }^{10}$. A atuação do enfermeiro, enquanto profissional de saúde, é preponderante no desenvolvimento de ações de conscientização e de difusão de informação entre os agricultores, além do fornecimento das orientações, bem como a assistência, o suporte e os cuidados necessários nos casos de intoxicação por agrotóxicos, contribuindo, assim, para a redução da ocorrência de malformações congênitas resultantes da exposição aos agrotóxicos"1.

Ao se considerar a carência de estudos nacionais sobre o tema, fica evidente a necessidade da realização de pesquisas de cunho epidemiológico, toxicológico ou estudos de caso controle, a fim de confirmar a associação da ocorrência de malformações fetais com a exposição aos agrotóxicos. Além disso, ao considerar que o município de estudo tem a sua economia baseada na agricultura, grande parte da população tem contato direto ou indireto com agrotóxicos, e se tornam relevantes as pesquisas que analisam o impacto dos agrotóxicos sobre a população exposta. Assim, o presente estudo tem como objetivo relacionar o uso de agrotóxicos com a ocorrência de malformações congênitas no município de Giruá (RS), bem como determinar os fatores de risco associados.

\section{Material e métodos}

A pesquisa é de abordagem quantitativa, do tipo descritivo-exploratória e foi realizada no município de Giruá, que fica situado na região noroeste do estado do Rio Grande do Sul. A coleta de dados ocorreu durante o primeiro semestre de 2019, no período de fevereiro a maio. O estudo foi conduzido em duas etapas: (a) associação da ocorrência de malformações com o uso de agrotóxicos; (b) determinação dos fatores de risco associados. 


\section{Associação da ocorrência de malformações com o uso de agrotóxicos}

Para determinar a associação do uso de agrotóxicos com a ocorrência de malformações congênitas no município de Giruá, primeiramente, foi realizado o levantamento das informações dos nascidos vivos com malformações, no período de 1999 a 2016. Tais dados foram obtidos do Sinasc, do Ministério da Saúde. Trata-se de um sistema de informação de base populacional, que agrega os registros contidos na declaração de nascidos vivos, o que permite diversas análises na área de saúde materno-infantil.

Foram desconsiderados os casos de nascidos vivos com registro ignorado ou desconhecido. As taxas de malformações foram calculadas por meio da seguinte fórmula:

$N^{o}$ de nascidos vivos com malformação $\times 1.000$

Total de nascidos vivos no período

Foi feita uma comparação das taxas de malformações ocorridas no município de Giruá, dividindo-se os dados em dois períodos: primeiro período (1999-2007) e segundo período (2008-2016). As taxas referentes ao segundo período foram tidas como referência, uma vez que apresentavam um menor nível de exposição. A possível associação do uso de agrotóxicos com a ocorrência de malformações no município foi calculada por meio do Odds Ratios (OR), e o Intervalo de Confiança (IC) adotado para as amostras foi de $95 \%$.

Os Odds Ratios fornecem dados sobre a força da associação entre o fator estudado e o desfecho, permitindo que se faça um julgamento sobre uma relação de causalidade. Para a construção da variável de exposição, levou-se em consideração a quantidade de lavouras plantadas por hectare de acordo com o ano de plantio, no período de 1999 a 2016. Foram selecionados três grãos de cultivo, principais commodites agrícolas da região, sendo estes: milho, soja, trigo. Os dados sobre plantio foram obtidos por intermédio do Instituto Brasileiro de Geografia e Estatística ${ }^{12,13}$.

\section{Determinação dos fatores de risco associados}

Para essa determinação, foi realizada uma entrevista com agricultores residentes no município de Giruá. A população rural do município é de, aproximadamente, 4.168 pessoas, o que indica uma margem de $24,4 \%$ dos habitantes do município, distribuídos em 1.440 propriedades rurais ${ }^{\mathbf{1 2}}$.

A população que serviu de base para a definição do tamanho da amostra foi de 1.440 indivíduos, por estarem de acordo com os seguintes critérios de inclusão: trabalhar na atividade agrícola, realizar manejo de agrotóxicos, residir na área rural do munícipio de Giruá e ser maior de 18 anos. Considerando essa população, foi calculado o tamanho amostral para planejamento da média numérica de pessoas que deveriam ser entrevistadas, sendo utilizada a seguinte fórmula ${ }^{14}$ :

$$
n=\frac{N \cdot Z^{2} \cdot p \cdot(1-p)}{Z^{2} \cdot p \cdot(1-p)+e^{2} \cdot(n-1)}
$$

Legenda: $\mathrm{n}$ - amostra calculada; $\mathrm{N}$ - população; Z - variável normalmente padronizada associada ao nível de confiança; p - verdadeira probabilidade do evento; e erro amostral.

Considerando um nível de confiança de $99 \%$ e um percentual máximo de $60 \%$ da população amostrada, foram entrevistados 63 produtores rurais.

A coleta de dados foi realizada por meio de um formulário estruturado com questões objetivas, abordando aspectos como: o uso de agrotóxicos, perfil sociodemográfico, conhecimento dos agricultores a respeito dos riscos de intoxicações, consequências para a saúde relacionadas à sua exposição, uso de equipamentos de proteção, sinais e sintomas característicos do contato com agrotóxicos, armazenamento e destino das embalagens vazias. 
A pesquisa foi realizada nas residências dos entrevistados. O pesquisador, após se apresentar, explicou o objetivo do trabalho, entregando o Termo de Consentimento Livre e Esclarecido, que o entrevistado, ao aceitar participar da pesquisa, assinou e recebeu uma cópia. Em seguida, o participante respondeu ao formulário acima proposto. Não houve recusa de nenhum dos participantes definidos na amostragem de participar da pesquisa, nem perda de amostra.

A apresentação dos dados foi feita em gráficos e tabelas. Para análise dos dados, foram construídas planilhas no Microsoft Excel ${ }^{\circledR}$. Foram formuladas tabelas de frequências (absoluta, relativa e relativa acumulada), IC de $95 \%$ e Odds Ratios. As associações entre as variáveis independentes com a variável dependente foram estabelecidas sendo utilizados os testes de razão de chances com o respectivo IC de $95 \%$ para as variáveis categóricas. Foi adotado o nível de significância de $5 \%$ em todos os testes.

A pesquisa foi submetida ao Comitê de Ética em Pesquisa (CEP) da Universidade de Passo de Fundo (UPF), no Rio Grande do Sul (RS), obtendo parecer favorável para a coleta de dados sob Certificado de Apresentação para Apreciação Ética (CAAE) $\mathrm{n}^{\circ}$ 98059118.5.0000.5342.

Os materiais com as informações obtidas para realização da pesquisa incluíram um Termo de Consentimento Livre e Esclarecido, conforme preconiza a Resolução no 466/12 do Conselho Nacional de Saúde/Ministério da Saúde (CNS/MS) ${ }^{15}$.

\section{Resultado e discussão}

Com relação à área plantada em hectares e às três principais culturas produzidas no município de Giruá, no período de 1999 a 2016, observou-se que, ao longo do período avaliado, houve aumento significativo na área cultivada. Em 1999, essa era de 68.100 hectares, já em 2016 foi de 92.450 hectares, o que representa um aumento de $26,34 \%$ observado nas três culturas consideradas (tabela 1).

Tabela 1. Quantitativo de hectares plantados na produção de milho, soja e trigo no município de Giruá, de 1999 a 2016. Giruá (RS), 2019

\begin{tabular}{lrrrr}
\hline Ano & \multicolumn{3}{c}{ Produto das lavouras temporárias } & Total (ha) \\
\hline 1999 & Milho & Soja & Trigo & 68100 \\
2000 & 2600 & 56500 & 9000 & 74000 \\
2001 & 3500 & 56500 & 14000 & 76500 \\
2002 & 6000 & 56500 & 14000 & 80200 \\
2003 & 3700 & 56500 & 20000 & 90700 \\
2004 & 3700 & 57000 & 30000 & 92000 \\
2005 & 3000 & 57000 & 32000 & 77000 \\
2006 & 2000 & 45000 & 30000 & 73000 \\
2007 & 5500 & 57000 & 10500 & 85500 \\
2008 & 5500 & 57000 & 23000 & 86300 \\
2009 & 4300 & 57000 & 25000 & 82500 \\
2010 & 3000 & 57000 & 22500 & 86000 \\
2011 & 5000 & 58000 & 23000 & 90500 \\
2012 & 4500 & 58000 & 28000 & 90300 \\
\hline
\end{tabular}


Tabela 1. (cont.)

\begin{tabular}{lrrrr}
\hline Ano & \multicolumn{3}{c}{ Produto das lavouras temporárias } \\
& Milho & Soja & Trigo & Total (ha) \\
\hline 2013 & 4300 & 58000 & 30000 & 92300 \\
2014 & 4500 & 60000 & 35000 & 99500 \\
2015 & 4600 & 63700 & 25000 & 93300 \\
2016 & 4600 & 63850 & 24000 & 92450 \\
\hline Total (ha) & & & & $\mathbf{1 5 3 0 1 5 0}$ \\
\hline
\end{tabular}

Destaca-se, ainda, no ano de 2016, que $68,77 \%$ da área cultivada no município eram destinados para a produção de soja. A segunda cultura de maior relevância para o município é o milho, que representa $25,96 \%$ da área produzida, e o restante é destinado ao cultivo de trigo (tabela 1).

A tabela 2 expressa a Odds Ratios Bruta e Intervalos de Confiança de nascidos vivos com malformações congênitas para o município de Giruá no período entre 1994 e 2016.

As análises das taxas de malformações congênitas foram divididas em dois períodos, 1999-2007 e 2008-2016, sendo de 17,90 e 15,08, respectivamente, totalizando uma taxa média de 16,74 para os dois períodos. A maior taxa de malformações congênitas foi encontrada no primeiro período. Já para a Unidade da Federação e para o estado do Rio Grande do Sul, as taxas de malformações congênitas neste período apresentaram uma média de 6,45 e 8,21 , respectivamente, ou seja, menor que no município analisado ${ }^{8}$.

Quanto aos Odds Ratios Bruta e Intervalos de Confiança de nascidos vivos com malformações congênitas para o município de Giruá, a maior relação encontrada refere-se à anomalia congênita, classificada como: 'Testículo não descido' (OR = 5,6, IC95\% = 3,85-10,41). Estudo realizado por Dutra; Ferreira ${ }^{5,6}$, nos estados do Paraná e de Minas Gerais, no período de 1994-2014, também relatou que a malformação de testículo não descido foi a que mais sobressaiu $(\mathrm{OR}=6,64, \mathrm{IC} 95 \%=3,85-11,46)$, assim como neste trabalho. Sobre esse aspecto, os agrotóxicos são suspeitos de influenciar a diferenciação sexual do feto e desfechos pendentes de hormônios sexuais ${ }^{16}$. Por serem disruptores endócrinos, sugere-se que os agrotóxicos são capazes de induzir à malformação de testículos não descidos ${ }^{17}$.

A segunda maior relação encontrada para o município refere-se às 'Malformações Congênitas do Aparelho Circulatório' ( $\mathrm{OR}=$ $4,21$, IC95\% $=4,21-6,01)$. Tal fato corrobora a pesquisa de Dutra; Ferreira ${ }^{5}$, em que a referida malformação obteve maior OR (OR $=5,32$, IC95\% = 4,48-6,31). Já em estudo realizado por Dutra; Ferreira ${ }^{6}$, no estado de Minas Gerais, as malformações do aparelho circulatório apresentaram a terceira maior relação $(\mathrm{OR}=3,27$, IC95\% $=2,83-3,77$ ).

A respeito dessas malformações, Carmen et al. 18 afirmam que os estados brasileiros com maior autoconsumo de agrotóxicos, nos anos 2013 e 2014, apresentaram aumento de 100 a $75 \%$ no risco de ocorrência de malformações congênitas do sistema cardiovascular, aspecto que é reforçado por estudo de caso controle realizado por Rocheleau et al. ${ }^{19}$. Ambos encontraram relação entre malformações congênitas cardíacas com a exposição ocupacional de mães a agrotóxicos, como alterações de septo atrial, estenose da válvula pulmonar, síndrome da hipoplasia do coração esquerdo.

Observou-se, ainda, que a terceira maior relação encontrada para o município de Giruá foi referente às 'Malformações Congênitas do 
Aparelho Osteomuscular' (OR = 3,56, IC95\% $=3,42-3,88)$. Dutra; Ferreira ${ }^{5}$, avaliando os casos encontrados no estado do Paraná, observaram resultado semelhante, enquanto, em estudo realizado no estado de Minas Gerais ${ }^{6}$, esse tipo de malformação apresentou a quinta posição. Esses resultados são reafirmados por Kalliora ${ }^{20}$, que concluiu que há uma tendência positiva para uma relação entre a exposição ocupacional e o uso de alguns agrotóxicos com anomalias congênitas do sistema osteomuscular.

Destaca-se, ainda, a quarta maior relação encontrada no município, que se refere às 'Malformações Congênitas do Aparelho Geniturinário' (OR = 3,2, IC95\% = 2,66-3,46). Mais uma vez, esses resultados corroboram os obtidos por Dutra; Ferreira ${ }^{5}$, em que as malformações do aparelho geniturinário apresentaram a quarta maior relação $(\mathrm{OR}=3,28$, IC95\%2,86-3,75). Já em pesquisa realizada por Dutra; Ferreira ${ }^{6}$, no estado de Minas Gerais, ela ficou na sexta posição (OR = 2,60, IC95\% 2,34-2,88), comprovando a relação do uso de agrotóxicos com esse tipo de malformação, o que foi evidenciado, ainda, por Kalliora ${ }^{20}$, que afirmou haver relação entre exposição ambiental e ocupacional no uso de agrotóxicos e anomalias congênitas do sistema urogenital.

As demais relações encontradas nas malformações congênitas são: Outras Malformações Congênitas ( $\mathrm{OR}=2,9, \mathrm{IC}=2,60-3,44)$, outras Malformações Congênitas do Aparelho Digestivo ( $\mathrm{OR}=2,75, \mathrm{IC}=2,43-3,25)$ e Anomalias Cromossômicas NCOP (OR $=2,63, \mathrm{IC}=2,40$ $3,10)$, respectivamente.

Estudo realizado por Toichuev $\mathbf{2 1}^{\mathbf{2 1}}$ verificou a presença de organoclorados em placentas de mulheres que moravam em área de maior exposição aos agrotóxicos, sendo observados problemas de saúde em recém-nascidos de baixo peso ao nascer, malformações congênitas, infecções e natimortos, em prematuro com parto prematuro, pré-eclâmpsia/gestacional e frequência de hospitalização após o parto. Os dados do estudo evidenciaram, mais uma vez, que há relação entre o uso de agrotóxicos e a ocorrência de malformações fetais, e que são necessárias campanhas de prevenção e de conscientização da população sobre esse tema.

Tabela 2. Número, Odds Ratios e Intervalos de Confiança de nascidos vivos com malformação congênita para o município de Giruá. Giruá (RS), 2019

\begin{tabular}{|c|c|c|c|c|c|}
\hline Tipo de Anomalia Congênita & 1999-2007 & 2008-2016 & Total & $\mathrm{OR}^{\star}$ & IC ${ }^{\star \star 9} 95 \%$ \\
\hline Malformações do Sistema Nervoso & 1 & 0 & 1 & 2,21 & $2,01-2,45$ \\
\hline Malformações do Aparelho Circulatório & 1 & 1 & 2 & 4,21 & $4,21-6,01$ \\
\hline Fenda labial, Fenda Palatina & 2 & 1 & 3 & 2,27 & $2,32-2,86$ \\
\hline Malformações do Aparelho Digestivo & 1 & 1 & 2 & 2,75 & $2,43-3,25$ \\
\hline Testículo não descido & 4 & 6 & 10 & 5,6 & $3,85-10,41$ \\
\hline Malformações do Aparelho Geniturinário & 4 & 2 & 6 & 3,2 & $2,66-3,46$ \\
\hline Deformidades do Quadril & 0 & 3 & 3 & 1,3 & $0,90-2,01$ \\
\hline Deformidades dos Pés & 8 & 2 & 10 & 2,5 & $2,35-2,78$ \\
\hline Malformações do Aparelho Osteomuscular & 5 & 6 & 11 & 3,56 & $3,42-3,88$ \\
\hline Outras Malformações Congênitas & 11 & 2 & 13 & 2,9 & $2,60-3,44$ \\
\hline Anomalias Cromossômicas NCOP & 4 & 0 & 4 & 2,63 & $2,40-3,10$ \\
\hline Total de nascidos vivos com malformações & 41 & 24 & 65 & 3,01 & $2,90-3,10$ \\
\hline Taxa de malformações congênitas & 17,90 & 15,08 & 16,74 & & \\
\hline
\end{tabular}


Percebe-se que os resultados encontrados no estudo corroboram outros estudos, com destaque para estudo realizado no Paraná ${ }^{5}$, em que as posições das malformações são idênticas, e os OR bem próximos aos obtidos neste estudo. Já o estudo realizado em Minas Gerais ${ }^{6}$ também apresenta resultados semelhantes, mas com algumas variações.

Para determinar os fatores de risco associados com a exposição aos agrotóxicos e, consequentemente, a possibilidade de malformações, foram entrevistados 63 agricultores, sendo eles proprietários e funcionários, maiores de 18 anos, que pertencem ao município de Giruá. A maioria com idade acima de 50 anos e do sexo masculino (tabela 3). A prevalência do sexo masculino na agricultura está relacionada ao fato de que o trabalho de preparação e de aplicação de agrotóxicos e outras atividades desenvolvidas na agricultura ainda são predominantemente masculinas, principalmente em propriedades que cultivam soja, trigo e milho, que são gerenciadas por homens, realidade que se apresenta em âmbito nacional22.

No contexto atual, as mulheres têm risco de exposição ocupacional aos agrotóxicos, uma vez que auxiliam no preparo dos produtos antes da aplicação, além de ajudarem puxando a borracha para pulverizar agrotóxicos no caso de equipamentos mecânicos, sendo que, na maioria dos casos, elas fazem esse trabalho sem quaisquer Equipamentos de Proteção Individual (EPIs) ${ }^{23}$. Além disso, existe o risco de exposição ambiental, por deriva técnica, quando residem em locais próximos às áreas de aplicação, e o risco de exposição paraocupacional, pois realizam lavagem das roupas contaminadas e ou dos EPIs ${ }^{24}$.

Destaca-se que uma das dificuldades em mensurar o impacto da exposição ocupacional por parte das mulheres ocorre em função do fato de que o trabalho rural muitas vezes não é considerado por elas como um trabalho, e sim um tipo de ajuda conferida aos seus parceiros, entendendo a atividade feminina como apenas complementar e contribuindo para a característica universal de segregação ocupacional ${ }^{25}$. Há maior risco de ocorrência de malformações congênitas entre mães que manuseiam roupas e ferramentas contaminadas trazidas pelos maridos ${ }^{8}$.

O estudo apontou um baixo grau de escolaridade entre os trabalhadores rurais do município, em que $61,90 \%$ possuem Ensino Fundamental incompleto, e uma pequena porcentagem de pessoas possui nível superior completo (tabela 3). O baixo nível de escolaridade é um dos fatores associados ao risco de intoxicações, pela dificuldade de ler e entender as instruções fornecidas nos rótulos dos agrotóxicos sobre o correto manuseio desses produtos ${ }^{26}$. O estudo de Silva et al. ${ }^{16}$ observou maior tendência de defeitos congênitos em neonatos de genitores com níveis baixos de escolaridade, sendo que, nos casos analisados, $71 \%$ dos pais e $64 \%$ das mães tinham nível de ensino fundamental incompleto, assim como o observado nesta pesquisa.

Por outro lado, os agricultores não devem ser culpabilizados pela sua baixa escolaridade e consequente intoxicação. Nesse contexto, a legislação prevê que, à direita do eixo central da faixa colorida dos rótulos de agrotóxicos, sejam colocados pictogramas referentes às precauções durante a aplicação, que devem ser de fácil entendimento. Essa seria uma alternativa para as pessoas de baixa escolaridade ${ }^{27}$.

Todos os entrevistados utilizam agrotóxicos nas lavouras, sendo que os agrotóxicos pertencentes às classes toxicológicas de cor vermelha (extremamente tóxicos) e amarela (altamente tóxicos) são os mais utilizados (tabela 4), o que representa um maior risco à saúde humana e ambiental. Na cultura de tomate, de pimentão e em pastagens, os agrotóxicos mais utilizados são os de tarjas vermelhas e amarelas (68\%), que apresentam grau altamente tóxico ${ }^{22}$. Destaca-se que agrotóxicos com maior grau de toxicidade têm maior probabilidade de estarem associados ao desenvolvimento de malformações fetais na população exposta. 
Tabela 3. Características sociodemográficas dos agricultores entrevistados. Giruá (RS), 2019

\begin{tabular}{lccr}
\hline Variáveis & No & Fr & Fra \\
\hline Idade & & & \\
\hline 18 a 30 anos & 7 & 11,11 & 10 \\
31 a 40 anos & 7 & 9,52 & 19,52 \\
41 a 50 anos & 43 & 11,11 & 30,63 \\
Mais de 50 anos & & 68,25 & 98,89 \\
\hline Sexo & 9 & 14,29 & 10 \\
\hline Feminino & 54 & 85,71 & 95,71 \\
Masculino & & & 10 \\
\hline Grau de Escolaridade & 0 & 0,00 & 71,90 \\
\hline Analfabeto & 39 & 61,90 & 78,25 \\
Ensino Fundamental incompleto & 4 & 6,35 & 86,19 \\
Ensino Fundamental completo & 5 & 7,94 & 94,13 \\
Ensino Médio incompleto & 5 & 7,94 & 102,06 \\
Ensino Médio completo & 5,94 & 110,00 \\
\hline Ensino Superior incompleto & 5 & 7,94 & \\
Ensino Superior completo & 5 & & \\
\hline Fonte: & & 7,94 & \\
\hline
\end{tabular}

Fonte: Elaboração própria.

A grande maioria dos entrevistados tem consciência dos efeitos e riscos que os agrotóxicos podem causar à saúde, como câncer e malformações congênitas. Entre os entrevistados, é expressivo o número de pessoas que afirmam já terem se intoxicado (30,16\%) com algum tipo de agrotóxico (tabela 4). Em estudo realizado por Silva et al. ${ }^{20}, 33,33 \%$ dos trabalhadores entrevistados relataram terem sido diagnosticados com intoxicação aguda, dos quais, $25 \%$ procuraram atendimento médico, enquanto $8,33 \%$ não procuraram nenhum atendimento.

Destaca-se que, no Brasil, é expressivo o número de intoxicações relacionadas à exposição e à utilização de agrotóxicos nas lavouras, e foram notificados pelo Ministério da Saúde, no período entre 2007 a 2014, um total de 25.106 mil casos de intoxicação por agrotóxicos ${ }^{28}$. Preocupa, ainda, a existência de grande número de subnotificações nos casos de intoxicação por agrotóxicos, o que é um agravante no contexto da saúde pública e um obstáculo ao trabalho de vigilância.
As causas de subnotificação podem estar relacionadas com as dificuldades de registro das informações, quando há ausência de relato de intoxicação pelo trabalhador rural por dificuldade de acessar um serviço de saúde, ou quando há atendimento, e os profissionais de saúde relacionam a sintomatologia com outras doenças e agravos ou quando os registros não são efetivados nos sistemas de informação ${ }^{29}$. Diante desse cenário de subnotificação, é fundamental que os profissionais de saúde saibam notificar os casos de intoxicação de quem trabalha em áreas rurais e, a partir disso, desenvolver e implementar estratégias de educação em saúde que contribuam para a redução dos riscos de intoxicação por agrotóxicos e para uma organização mais efetiva do atendimento dos serviços de saúde ${ }^{30}$.

Os demais entrevistados, mesmo sem apresentarem o diagnóstico, relataram sinais e sintomas após aplicação ou durante o manuseio com agrotóxicos, como: náusea, fraqueza muscular, vômito e cefaleia (tabela 4). Sintomas como mal-estar, fraqueza, vômitos e náusea 
podem aparecer durante o adoecimento de trabalhadores causado pela intoxicação por agrotóxicos $^{31,32}$. Pesquisa desenvolvida por Lopes; Albuquerque ${ }^{32}$ observou resultados semelhantes, em que os sintomas mais relatados pelos trabalhadores foram dores de cabeça, náuseas e dor de estômago, depressão, ansiedade e mialgia.

No que se refere aos princípios ativos mais utilizados entre os entrevistados, destacam-se: glifosato, paraquat, acefato, abamectina, 2,4 D Amina e atrazina, os quais são de classes toxicológicas extremamente e mediamente tóxicas (tabela 4). Entre os 504 princípios ativos autorizados no Brasil, 149 são proibidos na União Europeia, dos quais, dois estão entre os dez mais utilizados pelo Brasil (acefato é o $3^{\circ}$ mais usado, e o atrazina o $\left.7^{\circ}\right)^{28}$. O acefato pode causar prejuízos fetais ao sistema neurológico, imunológico, endócrino e reprodutor - neste último caso, podendo levar à ocorrência de malformações congênitas ${ }^{32}$.

O glifosato é largamente utilizado no Brasil, e a sua exposição pode causar sintomas como: convulsões, problemas respiratórios, hipertermia, perda de apetite, vômitos, náusea, sangramento nasal, desmaios. Quando a pessoa é exposta ao produto, por longo período de tempo, esse pode causar câncer, lesões no fígado, no pulmão, na pele e malformações congênitas ${ }^{33,34}$.

Tabela 4. Conhecimento dos agricultores entrevistados relacionado aos agrotóxicos, intoxicação, classe toxicológica, princípios ativos. Giruá (RS), 2019

\begin{tabular}{|c|c|c|c|}
\hline Variáveis & № & $\mathrm{Fr}$ & Fra \\
\hline \multicolumn{4}{|l|}{ Utiliza Agrotóxicos } \\
\hline $\operatorname{Sim}$ & 63 & 100 & 10 \\
\hline Não & 0 & 0 & 10 \\
\hline \multicolumn{4}{|l|}{ Classe Toxicológica } \\
\hline CI - Vermelho - Extremamente Tóxico & 49 & 77,78 & 10 \\
\hline C II - Amarelo - Altamente Tóxico & 53 & 84,13 & 94,127 \\
\hline C III - Azul - Mediamente Tóxico & 42 & 66,67 & 160,79 \\
\hline CIV - Verde - Pouco Tóxico & 9 & 14,29 & 175,08 \\
\hline \multicolumn{4}{|l|}{ Intoxicação } \\
\hline Sim & 19 & 30,16 & 10 \\
\hline Não & 44 & 69,84 & 79,84 \\
\hline \multicolumn{4}{|l|}{ Consciência dos riscos dos agrotóxicos } \\
\hline Sim & 62 & 98,41 & 10 \\
\hline Não & 1 & 1,59 & 11,59 \\
\hline \multicolumn{4}{|l|}{ Sinais/Sintomas } \\
\hline Náusea & 15 & 23,81 & 10 \\
\hline Alergia de pele & 6 & 9,52 & 19,52 \\
\hline Fraqueza muscular & 11 & 17,46 & 36,98 \\
\hline Cefaleia & 9 & 14,29 & 51,27 \\
\hline Vômito & 16 & 25,40 & 76,67 \\
\hline Ardência nos olhos & 4 & 6,35 & 83,02 \\
\hline Outros & 7 & 11,11 & 94,13 \\
\hline
\end{tabular}


Tabela 4. (cont.)

\begin{tabular}{lcrr}
\hline Variáveis & No & Fr & Fra \\
\hline Malformações Fetais e Câncer & & & \\
\hline Tem ciência & 61 & 96,83 & 10 \\
Não tem ciência & 2 & 3,17 & 13,17 \\
\hline Princípios Ativos & 27 & 42,86 & 10 \\
\hline Abamectina & 62 & 98,41 & 108,41 \\
Glifosato & 37 & 58,73 & 167,14 \\
Paraquat & 28 & 44,44 & 211,59 \\
Acefato & 22 & 34,92 & 246,51 \\
Atrazina & 2 & 3,17 & 249,68 \\
Tiametoxam & 26 & 41,27 & 290,95 \\
2-4 D Amina & 13 & 20,63 & 311,59 \\
Carboxina & 4 & 6,35 & 317,94 \\
Clorantraniliprole & & &
\end{tabular}

Fonte: Elaboração própria.

Sobre o local adequado para armazenamento dos agrotóxicos, a maioria dos entrevistados diz ter um local adequado para o seu acondicionamento - em um local separado dentro de um galpão (tabela 5). Essa situação foi observada em estudo de Sousa et al.27, segundo o qual a maioria dos agricultores relatou armazenar esses produtos em armazéns (70\%), sendo que $13 \%$ guardam em casa e $17 \%$ armazenam no campo.
Com relação ao destino das embalagens vazias, a maioria dos agricultores faz o destino correto das embalagens, entregando-as em uma empresa de reciclagem com prévio agendamento de horário para entrega (tabela 5), em cumprimento à Lei $\mathrm{n}^{0}$ 9.974, de 06 de junho de 2000, que dispõe sobre a produção, a embalagem e a rotulagem, o transporte, o armazenamento, a comercialização, a utilização e a fiscalização de agrotóxicos ${ }^{35}$.

Tabela 5. Local adequado para depósito, frequência de uso dos agrotóxicos, uso de EPIs. Giruá (RS), 2019

\begin{tabular}{lcrr}
\hline Variáveis & № & Fr & Fra \\
\hline Local adequado para depósito & & & \\
\hline Sim & 41 & 65,08 & 10 \\
Não & 22 & 34,92 & 44,92 \\
\hline Destino das embalagens vazias & 60 & 95,24 & 10 \\
\hline Devolvidas no estabelecimento & 3 & 4,76 & 14,76 \\
Outros & & & 10 \\
\hline Frequência de uso dos agrotóxicos & 1 & 1,59 & 14,76 \\
\hline Diariamente & 3 & 4,76 & 76,67 \\
Semanalmente & 39 & 61,90 & 103,65 \\
Mensalmente & 17 & 26,98 & 108,41 \\
\hline Período de ciclo da cultura & 3 & 4,76 & \\
Eventualmente & & & 10 \\
\hline
\end{tabular}


Tabela 5. (cont.)

\begin{tabular}{lccr}
\hline Variáveis & No & Fr & Fra \\
\hline Faz uso de EPIs & & & \\
\hline Sim & 42 & 66,67 & 10 \\
Não & 21 & 33,33 & 43,33 \\
\hline Quais EPIs & & & \\
\hline Luvas & 39 & 61,90 & 10 \\
Máscara & 38 & 60,32 & 70,317 \\
Botas & 27 & 42,86 & 113,17 \\
Roupas de proteção & 21 & 33,33 & 146,51 \\
Óculos & 7 & 11,11 & 157,62 \\
Outros & 1 & 1,59 & 159,21 \\
\hline
\end{tabular}

Fonte: Elaboração própria.

Com relação à periodicidade de aplicação dos produtos, a frequência mensal foi a que mais se destacou, seguida da aplicação por período de ciclo da cultura (tabela 5).

Entre os agricultores entrevistados, 33,33\% não utilizam EPIs, e os que utilizam não o fazem de maneira adequada, priorizando um ou mais equipamentos que consideram mais importantes e mais cômodos. Os EPIs mais utilizados são as luvas, máscaras e botas, e os óculos surgem como o EPI menos utilizado (tabela 5). Estudos observaram que a maioria dos agricultores não usa EPI pelo incômodo, por negligência ou uso inadequado, corroborando este estudo ${ }^{28-31}$. Somam-se a isso as orientações sobre uso de EPIs, os rótulos e as bulas dos agrotóxicos, que possuem letras com fontes pequenas, dificultando a leitura.

Nesse contexto, é evidente que os agricultores entrevistados estão expostos aos fatores de risco associados à ocorrência de intoxicação por agrotóxicos e, por consequência, à possibilidade de ocorrência de malformações congênitas.

Dessa forma, o profissional de saúde pode auxiliar na redução da exposição aos fatores de risco, atuando no planejamento de ações e estratégias que possibilitem o estabelecimento de um diálogo com os agricultores, sob o aspecto da educação e da informação ${ }^{10}$.
Ressalta-se que o profissional da enfermagem, enquanto membro da equipe de saúde, deve atuar nos diversos níveis de atenção, tanto na administração dos serviços quanto na assistência da população, de forma que o instrumento que se faz muito importante é o conhecimento acerca dos agravos relacionados aos agrotóxicos que podem acometer a saúde da população $0^{36}$.

Assim, o fortalecimento de ações de Vigilância em Saúde se torna indispensável para a formulação de estratégias de prevenção e da assistência, com base na identificação e na notificação dos casos de intoxicações por agrotóxicos. Diante disso, destaca-se a importância dos profissionais de saúde, especialmente a de qualificação dos enfermeiros, para orientarem os usuários do serviço, atentarem aos sinais e sintomas decorrentes da aplicação de agrotóxicos e realizarem a notificação em casos de intoxicação ${ }^{37}$.

\section{Considerações finais}

Os resultados da presente pesquisa convergem com numerosos estudos que comprovam os graves danos à saúde provocados pelos agrotóxicos. As malformações congênitas são um importante problema de saúde pública, 
sobretudo em regiões nas quais predominam a agricultura e o uso de agrotóxicos, como o município de estudo. Percebe-se que as taxas de malformações congênitas $(16,74)$ do município de Giruá são superiores à média do estado para o mesmo período.

Há a probabilidade de que a ocorrência de malformações no munícipio esteja associada ao uso de agrotóxicos, uma vez que todos os valores observados para os Odds Ratios foram maiores que um, sendo as maiores relações referentes às malformações classificadas como: Testículo não descido, Malformações Congênitas do Aparelho Circulatório, Malformações Congênitas do Aparelho Osteomuscular e Malformações Congênitas do Aparelho Geniturinário, respectivamente. Esses resultados corroboram os obtidos em outros estudos realizados por Oliveira et al. ${ }^{9}$, Dutra e Ferreira ${ }^{5,6}$, Asmus et al. ${ }^{38}$.

Neste estudo, foram evidenciados os seguintes fatores de risco associados com a possibilidade de intoxicação por agrotóxicos e a ocorrência de malformações congênitas: 0 baixo nível de escolaridade dos agricultores, o grau de toxidade e os princípios ativos dos agrotóxicos utilizados, o número de pessoas diagnosticadas com intoxicação, ou que apresentaram algum sinal e/ou sintoma de intoxicação, periodicidade das aplicações e não uso dos EPIs de maneira adequada.

Ressalta-se a importância de prevenção e controle do uso de agrotóxicos, já que o uso indiscriminado causa danos tanto para o meio ambiente como para trabalhadores e indivíduos envolvidos nesse contexto. Em função da gravidade deste tema, é importante identificar, de forma exclusiva, aspectos que envolvam a atuação dos profissionais de enfermagem para a melhoria da qualidade de vida e saúde dessas pessoas.

\section{Colaboradores}

Ferreira LF (0000-0001-7120-0171)*, ReolonCosta A (0000-0002-8160-9373)* e Ceolin S (0000-0001-6635-5515)* contribuíram igualmente para a elaboração do manuscrito.

\section{Referências}

1. Costa MGSG, Dimenstein MDB, Leite JF. Condições de vida, gênero e saúde mental entre trabalhadoras rurais assentadas. Rev. Estud Psicol. 2014 set [acesso em 2020 maio 12]; 19(2):145-54. Disponível em: http://www.scielo.br/scielo.php?pid=S1413$-294 X 2014000200007 \&$ script=sci_arttext\&tlng=pt.

2. Martins MA. O trabalhador rural e os agrotóxicos.
Rev. Dir. Trab. Polít. Soc. [internet]. 2015 [acesso em 2019 maio 1]; 1(1):198-207. Disponível em: http://revista91.hospedagemdesites.ws/index.php/rdtps/article/view/10.

3. Santos VCF, Trevilato GC, Mesquita MO, et al. O uso de agrotóxicos e suas implicações para a saúde humana e ambiental: enfrentamento local. Rev. Lume. [in-
*Orcid (Open Researcher and Contributor ID). 
ternet]. 2018 [acesso em 2020 maio 12]; 1(1):181-195. Disponível em: https://www.lume.ufrgs.br/bitstream/handle/10183/184491/001079419.pdf?sequence=.

4. Cabral ERM, Alonzo HGA. Aumento das exposições aos agrotóxicos: contribuição da enfermagem. Rev. Enfer. Atual. Inder. [internet]. 2019 [acesso em 2019 maio 9]; 87(2):87-8. Disponível em: https://revistaenfermagematual.com.br/index.php/revista/article/view/207.

5. Dutra LS, Ferreira AP. Associação entre malformações congênitas e a utilização de agrotóxicos em monoculturas no Paraná, Brasil. Saúde debate [internet]. 2017 [acesso em 2019 mar 12]; 2(41):241-253. Disponível em: http://www.scielo.br/pdf/sdeb/v41nspe2/01031104-sdeb-41-spe2-0241.pdf.

6. Dutra LS, Ferreira AP. Malformações congênitas em regiões de monocultivo no estado de Minas Gerais, Brasil. Rev. Med. (Ribeirão Preto) [internet]. 2017 [acesso em 2019 abr 5]; 50(5):285-96. Disponível em: http://dx.doi.org/10.11606/issn.2176-7262. v50i5p285-296.

7. Brasil. Ministério da Saúde. DATASUS: Sistema de Informações Sobre Nascidos Vivos - SINASC. Anomalia Congênitas. MS/SVS/DASIS [internet]. 2018 [acesso em 2019 mar 5]. Disponível em: http://tabnet.datasus.gov.br/cgi/deftohtm.exe?sinasc/cnv/nvbr. def.

8. Rigotto RM, Silva AMC, Ferreira MJM, et al. Tendências de agravos crônicos à saúde associados a agrotóxicos em relação de fruticultura no Ceará, Brasil. Rev. Brasil. Epidem. [internet]. 2013 [acesso em 2019 mar 20]; 16(3):763-773. Disponível em: http://dx.doi. org/10.1590/S1415-790X2013000300019.

9. Oliveira NM, Moi GP, Santos MA, et al. Malformações congênitas em municípios de grande utilização de agrotóxicos em Mato Grosso. Ciênc. Saúde. Colet. [internet]. 2014 [acesso em 2019 maio 08]; 1(1):41234129. Disponível em: http://www.scielo.br/scielo.php ?pid=S141381232014001004123\&script=sci_abstract.

10. Machado LM, Beck CL, Coelho APF, et al. Atuação dos profissionais de saúde da família frente ao trabalhador rural exposto a agrotóxicos. Rev. Cien. Cuid. Saúde [internet]. 2017. [acesso em 2020 maio 12]; 16(3):1-8. Disponível em: http://periodicos.uem.br/ ojs/index.php/CiencCuidSaude/article/view/37051.

11. Martins HV, Carvalho ABC, Belfort LRM, et al. O papel da enfermagem do trabalho na prevenção de riscos dos trabalhadores expostos aos agrotóxicos: uma revisão bibliográfica. Rev. Res. Soc. Dev. [internet]. 2010 [acesso em 2020 maio 12]; 8(6):1-12. Disponível em: https:// www.researchgate.net/publication/332118321_O_papel_da_enfermagem_do_trabalho_na_prevencao_de_ riscos_dos_trabalhadores_expostos_aos_agrotoxicos.

12. Instituto Brasileiro de Geografia e Estatísticas. Produção Agrícola Municipal. Tabela 1612. Área Plantada, área colhida, quantidade produzida [internet]. 2018. [acesso em 2019 abr 10]. Disponível em: https:// sidra.ibge.gov.br/Tabela/1612\#notas-tabela.

13. Instituto Brasileiro de Geografia e Estatísticas. Infográficos: evolução populacional e área da unidade territorial [internet]. 2018. [acesso em 2019 mar 15]. Disponível em: https://cidades.ibge.gov.br/brasil/rs/ girua/panorama.

14. Santos GEO. Cálculo Amostral: calculadora online [internet]. 2018. [acesso em 2019 mar 12]. Disponível em: http://www.calculoamostral.vai.la.

15. Conselho Nacional de Saúde. Resolução $n^{\circ}$ 466, de 12 de dezembro de 2012. Trata das diretrizes e Normas Regulamentadoras de Pesquisa envolvendo Seres Humanos. Brasília, DF: Ministério da Saúde [internet]. 2012. [acesso em 2019 abr 3]. Disponível em: https:// conselho.saude.gov.br/resolucoes/2012/Reso466.pdf.

16. Silva SRG, Martins JL, Seixas S, et al. Defeitos congênitos e exposição a agrotóxicos no Vale do São Francisco. Rev. Bras. Ginecol. Obstet. [internet]. 2011 [acesso em 2019 abr 15]; 33(1):20-26. Disponível em: http://www.scielo.br/pdf/rbgo/v33nl/a03v33nl.pdf.

17. Bueno PC. O impacto do consumo de agrotóxicos na prevalência de desfechos perinatais no Brasil. [dissertação] [internet]. Rio de Janeiro: Universidade Fe- 
deral do Rio de Janeiro; 2014. 89 p. [acesso em 2019 mar 8]. Disponível em: www.posgraduacao.iesc.ufrj. br/media/tese/1426601716.pdf.

18. Carmen IR, Camara VM, Raggio R, et al. Positive correlation between pesticide sales and central nervous system and cardiovascular congenital abnormalities in Brazil. Rev. Internat. Jour. Environ. Health. Research [internet]. 2017 [acesso em 2019 abr 20]; 27(1):2126. Disponível em: https://doi.org/10.1080/0960312 3.2017.1373272.

19. Rocheleau CM, Bertke SJ, Lawson CC, et al. Maternal occupational pesticide exposure and risk of congenital heart defects in the National Birth Defects Prevention Study. Rev. Birth. Defect. Research. Clin. Mol. Terat. [internet]. 2015 out [acesso em 2019 mar 20]; 103(10):823-33. Disponível em: https://www.ncbi. nlm.nih.gov/pmc/articles/PMC4607631.

20. Kalliora C, Manoulakis C, Vailopoulos E, et al. Association of pesticide exposure with human congenital abnormalities. Rev. Tox. Appl. Pharmacol [internet]. 2018 [acesso em 2019 mar 15]; 1(346):58-75. Disponível em: https://www.ncbi.nlm.nih.gov/pmc/articles/ PMC6029725/.

21. Toichuev RM, Zhilova LV, Paizildaev TR, et al. Organochlorine pesticides in placenta in Kyrgyzstan and the effect on pregnancy, childbirth, and newborn health. Rev. Environ. Sci. Pollut. Res. Int [internet]. 2018 [acesso em 2019 mar 15]; 1(32):31885-31894. Disponível em: https://www.ncbi.nlm.nih.gov/pubmed/29247409.

22. Vasconcelos MV, Freitas CF, Silveira CA. Caracterização do uso de agrotóxicos entre trabalhadores rurais. Rev. Saúde Santa Maria [internet]. 2014 jul/ dez [acesso em 2019 abr 9]; 40(2):87-96. Disponível em: http://periodicos.ufsm.br/revistasaude/article/ view/11934.

23. Leão RS, Marques RC, Buralli RJ, et al. Avaliação da saúde pública exposição a agroquímicos: uma experiência com a agricultura familiar o noroeste do Rio de Janeiro. Rev. Susten. em debate [internet] 2018 [acesso em 2020 maio 12]; 9(1):81-94. Disponível em:
https://scholar.google.com.br/scholar?hl=pt-BR\&as_ $\mathrm{sdt}=0 \% 2 \mathrm{C} 5 \&$ as_ylo=2016\&q=Avaliação+da+saúde+p ública+exposição.

24. Santana MC, Costa AR, Nunes MNF, et al. Exposição ocupacional de trabalhadores rurais a agrotóxicos. Rev. Cad. Saúde Colet. [internet] 2016 [acesso em 2020 maio 12]; 24(3):301-307. Disponível em: http:// www.scielo.br/scielo.php?pid=S1414462X20160003 00301\&script.

25. Lara TIC, Garcia SD. O impacto do uso dos agrotóxicos na saúde pública: revisão de literatura. Rev. Saúde e Desenv. Humano [internet] 2020 [acesso em 2020 maio 12]; 8(1):85-96. Disponível em: https://www. revistas.unilasalle.edu.br/index.php/saude_desenvolvimento/article.

26. Silva JV, Vilela LP, Moraes MS, et al. A percepção dos trabalhadores rurais sobre a autoexposição aos agrotóxicos. Rev. Saúde (Santa Maria) [internet]. 2017 [acesso em 2019 mar 20]; 43(1):199-205. Disponível em: https://periodicos.ufsm.br/revistasaude/article/ download/22163/15660.

27. Sousa JA, Feitosa HO, Carvalho CM, et al. Percepção dos produtores rurais quanto ao uso de agrotóxicos. Rev. Bras. Agricult. Irrig. [internet]. 2016 [acesso em 2019 mar 15]; 10(5):976-989. Disponível em: https://www. researchgate.net/publication/309643264_percepcao_ dos_produtores_rurais_quanto_ao_uso_de_agrotoxicos.

28. Silva JB, Xavier DS, Barboza MCN, et al. Fumicultores da zona rural de Pelotas (RS), no Brasil: exposição ocupacional e a utilização de equipamentos de proteção individual (EPI). Saúde debate [internet]. 2013 [acesso em 2019 mar 18]; 37(97):347-353. Disponível em: http://www.scielo.br/pdf/sdeb/v37n97/ v37n97al6.pdf.

29. Menck FW, Serafim MP, Oliviera JM. Intoxicação do(a) trabalhador(a) rural por Agrotóxicos: (sub) notificação e (in)visibilidade nas políticas públicas de 2001 a 2015. Rev. Segur. Aliment. Nutric. [internet] 2019 [acesso em 2020 maio 13]; (1)26:1-10. Disponível em: https://periodicos.sbu.unicamp.br/ojs/ index.php/san/article/view/8651513. 
30. Maia JMM, Lima JL, Rocha TJM, et al. Perfil de intoxicação dos agricultores por agrotóxicos em Alagoas. Rev. Diversitas Journal. [internet] 2018 [acesso em 2020 maio 13]; 3(2):486-504. Disponível em: https://periodicos.ifal.edu.br/diversitas_journal/article/view/626.

31. Cargnin MCS, Echer IC, Silva DR. Fumicultura: uso de equipamento de proteção individual e intoxicação por agrotóxico. Rev. Pesq. Cuidado. Fund. Online [internet]. 2017 [acesso em 2018 mar 10]; 9(2):466472. Disponível em: http://www.seer.unirio.br/index.php/cuidadofundamental/article/view/5444.

32. Lopes CVA, Albuquerque GSC. Agrotóxicos e seus impactos na saúde humana e ambiental: uma revisão sistemática. Saúde debate [internet]. 2018 [acesso em 2019 maio 4]; 42(117):518-534. Disponível em: http://dx.doi.org/10.1590/0103-1104201811714.

33. Bombardini LM. Atlas: Geografia do Uso de Agrotóxicos no Brasil e Conexões com a União Europeia [internet]. São Paulo: FFLCH-USP; 2017. [acesso em 2019 abr 22]. Disponível em: https://www.larissabombardi.blog.br/atlas2017.

34. Dutra RMS, Souza MMO. Impactos negativos do uso de agrotóxicos à saúde humana. Hygeia. Rev. Bras. Geog. Méd. Saúde [internet]. 2017 [acesso em 2019 mar 11]; 13(24):127-140. Disponível em: http://www. seer.ufu.br/index.php/hygeia.
35. Brasil. Lei $\mathrm{n}^{\circ}$ 9.974, de 6 de junho de 2000. Dispõe sobre a produção, destino, embalagens, transporte e armazenamento de agrotóxicos. República Federativa do Brasil [internet]. Diário Oficial da União. 7 Jun 2000. [acesso em 2019 mar 23]. Disponível em: http:/ planalto.gov.br/ccivil_03/leis/L9974.html.

36. Ambrosini MB, Witt RR. As Intoxicações por Agrotóxicos no meio rural e a atuação do enfermeiro. Rev. Gaúcha de Enfermagem [internet]. 2000 jan [acesso em 2019 fev 24]; 21(1):5-21. Disponível em: https:// seer.ufrgs.br/RevistaGauchadeEnfermagem/article/ view/4299.

37. Brasil. Ministério da Saúde. Protocolo de Atenção à Saúde dos Trabalhadores Expostos a Agrotóxicos [internet]. Brasília, DF: Ministério da Saúde; 2006 [acesso em 2019 mar 25]; 27 p. Disponível em: http:// bvsms.saude.gov.br/bvs/publicacoes/protocolo_atencao_saude_trab_exp_agrotoxicos.pdf.

38. Asmus FR, Camara VM, Raggio R, et al. Positive correlation between pesticide sales and central nervous system and cardiovascular congenital abnormalities in Brazil. Rev. Intern. Jour. of. Environ. Health. Res. [internet] 2016 [acesso em 2020 maio 12]; 5(1):420427. Disponível em: https://www.tandfonline.com/ doi/abs/10.1080/09603123.2017.1373272.

Recebido em 02/07/2019

Aprovado em 26/05/2020

Conflito de interesses: inexistente

Suporte financeiro: não houve 